\title{
TFT Substrate Glass Geometrical Parameter Measurement and Data Processing
}

\author{
H. Guo \\ Xi'an University of Technology \\ NO.5 South Jinhua Road, Xi’an, Shaanxi, China
}

\begin{abstract}
This paper has analyzed various sensors for the measurement of the TFT substrate glass geometric parameters and ascertain one of optical sensor for this task. The influence of measuring accuracy due to measuring platform has been studied, and then a solution for guarantee high precession measurement has been proposed.
\end{abstract}

Keywords-TFT glass; spectral confocal sensor; measuring platform

\section{INTRODUCTION}

There are many factors which influence the quality of TFT substrate glass and the LCD panel such as glass optical refractive index difference, manufactory stress and fault. The geometric parameters are also important factor, special the thickness and warpage. A new instrument consists of optical sensor, high-precision granite platform, CNC automatic controller was developed. It can measure the thickness, warpage, length and width, parallelism and perpendicularity of four edges of TFT substrate glass. The suitable sensor and the support platform on which the TFT substrate glass is lay when measuring is important faction for measuring accuracy. The study of material and the layout of the platform are presented in this paper.

\section{MEASURING TRANSDUCER}

The traditional measurement of glass thickness on the production line, including the detection of average thickness and the variation in thickness in both the flow and non-flow directions, is mostly done by the digital micrometer to manually measure the multi-point thickness on the glass edges, manually calculate the average thickness value and the variation in thickness in different directions. The thickness of TFT substrate glass is only $0.3-0.7 \mathrm{~mm}$, the required accuracy is high, in a large area of $2000 * 18000 \mathrm{~mm}$ of sixth generation version, the uncertainty for the thickness measurement should be controlled within $1.5 \mu \mathrm{m}$. The measuring speed of the traditional measuring methods is slow, and it is difficult to get the thickness value of whole part of glass. With the development of ultra-thin glass substrate towards the trend of new generation, the glass area is becoming larger and larger that it is more and more difficult for the thickness parameter of the edge part to reflect the thickness condition of the whole plate. The traditional method can no longer be used to handle the warpage measurement of substrate glass of large area.

New measuring instruments and equipment with different principles instead of the traditional mechanical measuring instruments in the glass thickness measurement field are studied, such as the ultrasonic thickness gauge, capacitance thickness gauge and various optical thickness gauges.

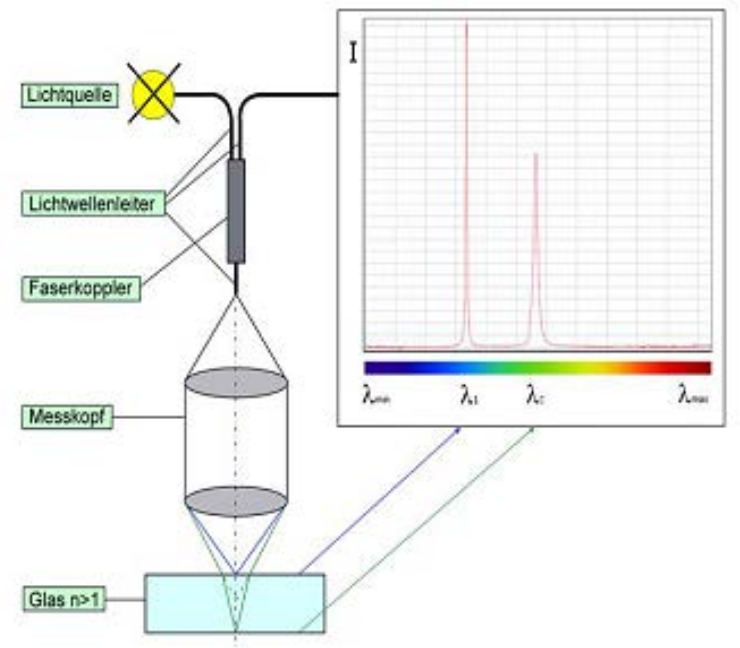

FIGURE I. PRINCIPLE OF SPECTRAL CONFOCAL MEASUREMENT.

The prime advantage of the optical measurement method is of its non-contact nature, which can avoid the precision loss caused by measuring force on the thickness, especially on the warpage. It can also realize the measurement of both the thickness and warpage simultaneously and improve the measurement efficiency. It can realize the CNC automatic measurement to conduct the objective assessment on the geometrical parameters of the whole plate by means high-speed and high-density sampling and data processing, so that optical sensor is very suitable for the measurement of TFT substrate glass. this paper has adopted the spectral confocal optical measurement sensor.

The spectral-confocal displacement sensor is developed based on in the confocal microscopy according the principle of spectral-confocal measurement. Its light source is not a single frequency laser, but the polychromatic light, after emitted, the polychromatic light shall go through the fiber coupler, after strict chromatic aberration correction through the lens group in the optical system, radiate the beam onto the surface to be measured to produce a spectral dispersion due to the action of color difference, forming a series of focus discrete in space. The monochromatic light focused on the object measured is reflected back to reach the sensor controller through optical fiber coupler, you can determine the frequency of the monochromatic light through the spectral analysis by 
spectrometer, as each frequency corresponds to a distance value, the displacement can be reckoned by the frequency so detected, so that you can accurately measure out the distance from the optical probe to the surface of object to be measured, of course, the refraction coefficient of the object to be measured can be substituted into the distance value in the calibration process.

If both the top and bottom surfaces of glass under test are placed within the measuring range of sensor probe, there shall have two different spectral peaks, $\lambda_{1}$ and $\lambda_{2}$, appeared on the spectrogram of the sensor detector, which correspond to the distances of two surfaces, $S_{1}$ and $S_{2}$, respectively.

The light ray shall enter into the top surface at an angle, if there is no refraction $(n=1)$, the beam shall intersect the optical axis at the point S0 away from the sensor. In case of refraction (the refractive index of glass usually $n>1$ ), the beam shall converge with the optical axis at a refraction angle $\beta$ at the point S2 away from the sensor.

According to the law of refraction, the relative refractive index $\mathrm{n}$ is a function of wavelength $\lambda$ :

$$
\sin \alpha / \sin \beta=n(\lambda)
$$

By the triangular relationship

$$
\frac{s_{2}-s_{1}}{s_{0}-s_{1}}=\frac{d_{2}}{d_{0}}=\frac{\tan \alpha}{\tan \beta}=\frac{\sin \alpha}{\sin \beta} \bullet \frac{\cos \beta}{\cos \alpha}=n \cdot \frac{\cos \beta}{\cos \alpha}
$$

Through comprehensive considerations of all the different beams, the real thickness $\mathrm{dw}$ can be determined by the measured thickness.

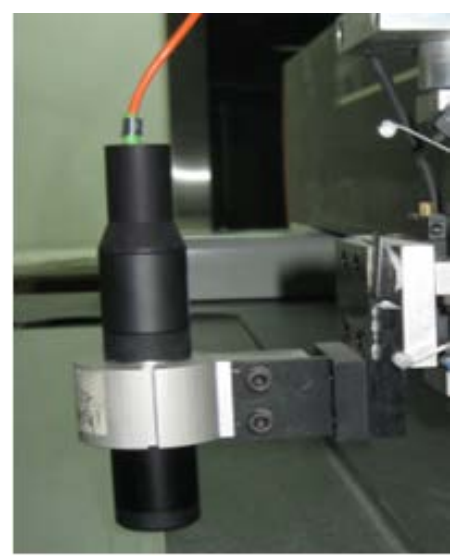

FIGURE II. EXPERIMENTAL INSTALLATION.

The spectral confocal sensor can measure the transparent or translucent workpiece with thin coating, as the light of different frequencies is reflected from the contact surface of different materials, therefore we can analyze on the distribution of spectrum detected by spectrometer to determine the thickness of each material layer through detecting the wavelength corresponding to each wave crest.
Based on this special optical principle and structural design, the spectral confocal sensor has a high accuracy in the measurement of displacement and thickness.

\section{IMPACT OF GLASS SUPPORT CONDITION ON MEASUREMENT}

As mentioned above, the measuring principle of sensor is based on the reflection of color light on the top and bottom surfaces of glass, when the glass is placed on different carriers, because the refractive index of supporting material itself, uniformity of material, state of contact interface and contact stress would affect the optical characteristics of the contact interface. The impact on the reflection on the top and bottom surfaces is different, which would greatly interfere the high precision measurement, produce the measurement noise and bring adverse influence to the measurement and evaluation. In order to study the impact of different supporting materials on the sensor, we select the optimum material to be used as the measuring platform.

In the experiment we placed the glass on different materials, such as rubber, plastic, granite and suspension (air) to measure same substrate glass sampled. The measurement results are shown in Figure 3. The abscissa represents the number of measuring points, while the ordinate represents the corresponding value of thickness. In order to reflect most of the measuring points, the range of thickness value on the ordinate is adaptively adjusted.

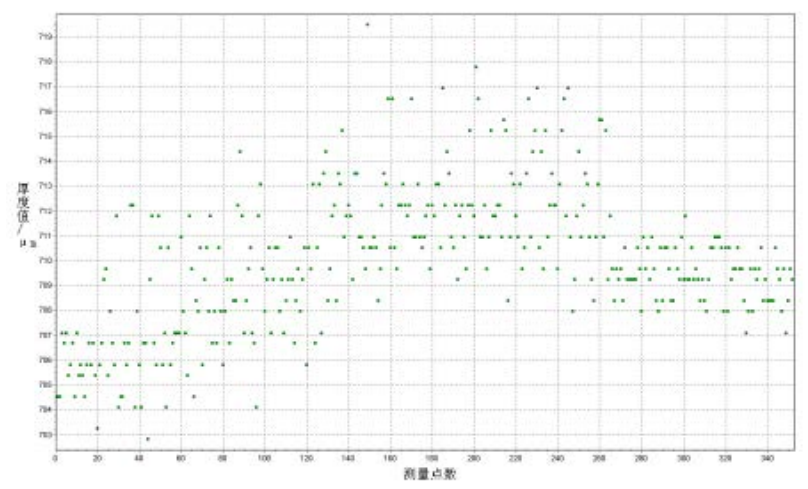

FIGURE III. (A) RUBBER.

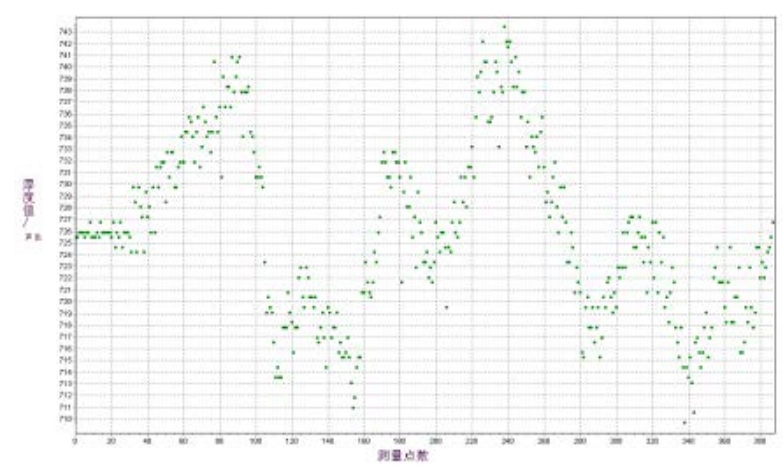

FIGURE III.(B) PLASTIC. 


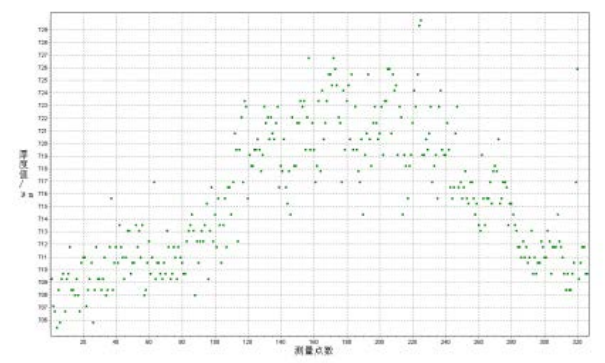

FIGURE III. (C) GRANITE.

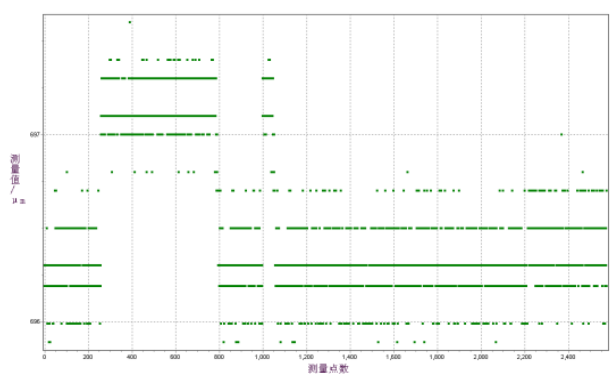

FIGURE III. (D) AIR.

The rubber surface is rougher that the diffuse reflection is stronger; the plastic surface is relatively rough, the reflectivity is moderate; because the surface of granite platform is finished, the surface is relatively smooth with a certain mirror reflection, and also with a certain degree of diffuse reflection.

It can be seen from the measuring point-thickness curve in the experiment, if the glass is placed on the surface of different carriers, the reflectivity of the carrier itself would affect the measurement results to some extent. The diffuse reflection of rubber is strong, there is no regular pattern to follow as to the impact on the measurement results. The impact of plastic carrier with moderate reflectivity on the measurement results is weaker in the regular pattern that the positions of the maximum and the minimum measured values have a certain repeatability, but the difference between the maximum and minimum values measured exceeds $30 \mu \mathrm{m}$, and the stability is poor. The impact of granite carrier on the measurement results is similar to plastic, the difference between the maximum and minimum

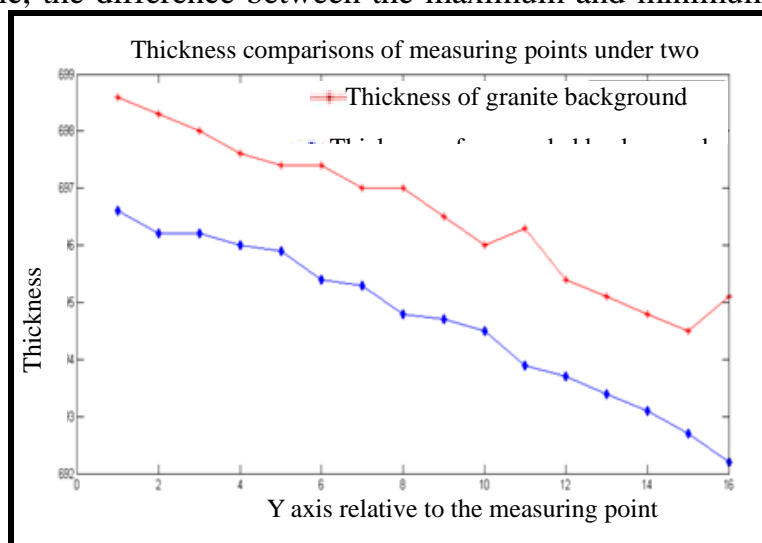

FIGURE IV. CONTRAST TEST OF DIFFERENT SUPPORTS. measured values is smaller, about $20 \mu m$, but because the distribution regularity of measuring points is not regular, it is impossible to be used to achieve the glass measurement.

The measuring point - thickness curve in the suspended state is shown as a line segment, the thickness value is mostly changing with the changes in the measuring points, the changes in the stable measurement data are within the $1.5 \mu \mathrm{m}$ linear deviation. Therefore, in order to ensure the stability of the measurement accuracy, it is necessary to measure the glass in the suspended state.

\section{Topology Optimization Design of MEAsuring PLATFORM}

In order to realize the warpage measurement of flat glass, there must have a measuring datum. Due to its excellent stability and vibration reducing performance, the granite is the preferred material for the mechanical measuring platform of large-sized precision measurement equipment. The automatic measuring equipment for TFT the substrate glass has adopted the granite platform, which is also used as the plane datum for the measurement of high precision guide and warpage pair in the measurement equipment. But when the granite material is used as the background of optical measurement, it would have an impact on the optical measurement.

In order to further study the impact of granite platform background on the results of the dynamic thickness measurement, it is necessary to make the further experimental researches. Fix the probe in the vertical direction of the glass plane and move along the glass plane direction, to write down the measurement results under the same high granite carrier background and suspended background respectively. Measure the thickness for 20 times under each of the two working conditions whenever the probe is moving a displacement $\mathrm{L}$ by the preset step length, and write down the mean value and standard deviation. The distribution of thickness values within the measuring range is shown Figure 4.

The distribution of standard deviation under the two carrier backgrounds is shown Figure 5.

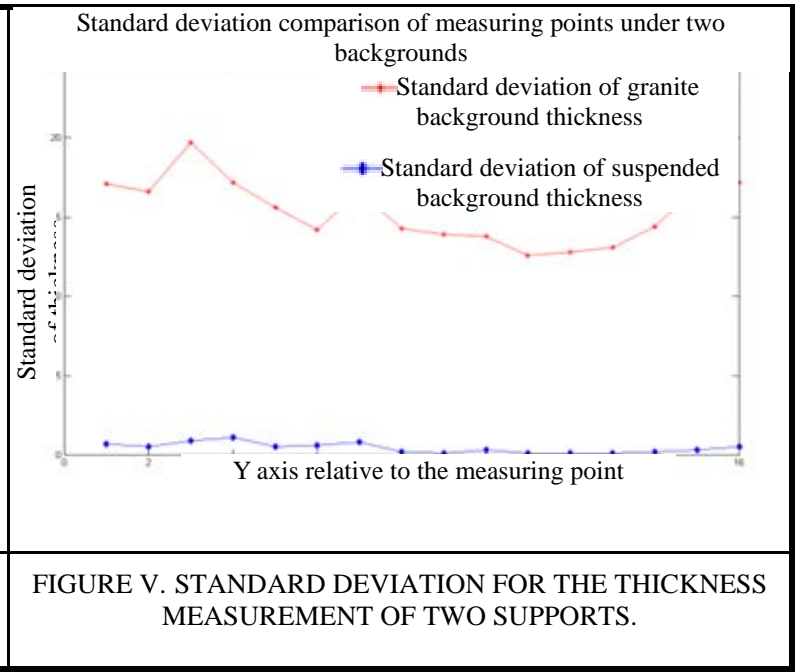


It is proved by the experimental analysis that, the difference in the measurement results under the granite background and suspended state is quite great, of the measurement under the granite carrier background, the distribution of measurement data is scattered, with great obviously random error; in contrast, with the dynamic measurement in the suspended state, the repeatability of measurement data is very good, the standard deviation is less than $1 \mu \mathrm{m}$, which can meet the thickness measurement requirements.

Therefore, in order to use the stable granite platform as the warpage measurement datum, while realizing the suspended measurement of ultra-thin substrate glass, this paper proposed to provide the equidistant measuring grooves in the platform along the non flow direction of TFT substrate glass, to avoid the impact of medium fluctuation between granite surface and glass interface on the probe data, which can also improve the measurement precision of TFT substrate glass thickness and warpage. Seeing from the angle of the glass thickness measurement, we hope the suspended glass area should be as large as possible. But for the glass with thickness to be $0.3-0.7 \mathrm{~mm}$, the plate support of equidistant grooves would produce additional deformation of thin glass. Therefore, the physical dimension of the groove section should be designed in a reasonable manner, the slot width should be as wide as possible under the condition that it would not produce the additional warpage deformation. In this paper we have
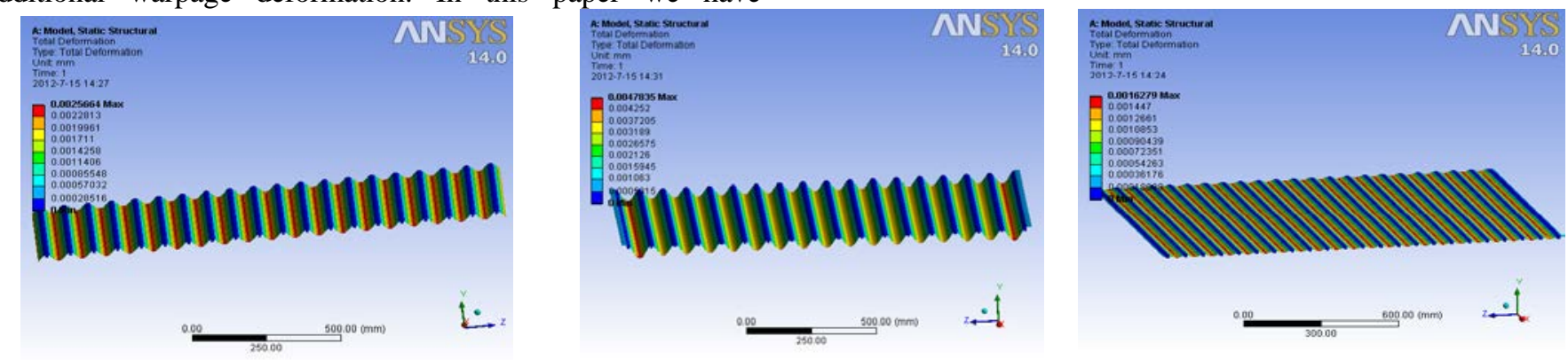

FIGURE VI. (A) CENTER DISTANCE OF SUPPORTS IS 90 MM, THE MAXIMUM DEFORMATION IS 4.9M. conducted analysis and experiment by the section design of supporting platform for the $2000 * 1800 \mathrm{~mm}$ glass with thickness to be $0.5 \mathrm{~mm}$.

In order to ensure the reliable operation of warpage and thickness optical measurement sensor, we should ensure the stability and reliability of original measured data. Based on the theoretical analysis and experimental results, the bottom surface of glass should be suspended for more than $3 \mathrm{~mm}$. By which the slots are opened in the granite measuring plate.

However, the width of slot and the shape of cross section shall have a certain impact on the glass warpage. In addition, the slot structure is closely related to the layout of measuring positions, wider the slot to open, the more favorable for the layout and arrangement of measuring positions shall be, but the wider slot would have more obvious impact on the glass warpage.

For this purpose, we conducted the in-depth analysis on the impact of slot structure on the warpage. For the TFT substrate glass with thickness to be $0.5 \mathrm{~mm}$ (with elastic modulus to be $4.62 * 109$, Poisson's ratio to be 0.245 and density to be 2500 ), there are multiple supporting ribs across the glass, the supporting spacing is ranged from $90 \mathrm{~mm}$ to $50 \mathrm{~mm}$ that the in-depth analysis is made under various changed conditions. The results are as follows.

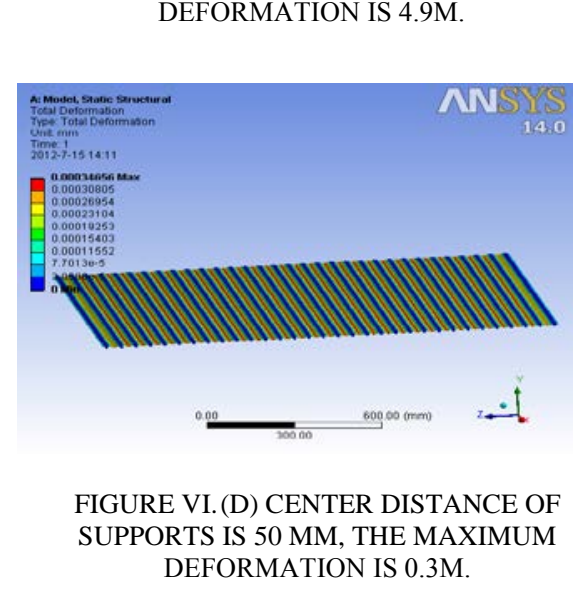

FIGURE VI.(D) CENTER DISTANCE OF SUPPORTS IS $50 \mathrm{MM}$, THE MAXIMUM DEFORMATION IS 0.3M.

The analysis results show that, for the $0.5 \mathrm{~mm}$ thick TFT substrate glass, smaller the support spacing is, the less impact
FIGURE VI.(B) CENTER DISTANCE OF SUPPORTS IS $80 \mathrm{MM}$, THE MAXIMUM DEFORMATION IS $2.6 \mathrm{M}$.

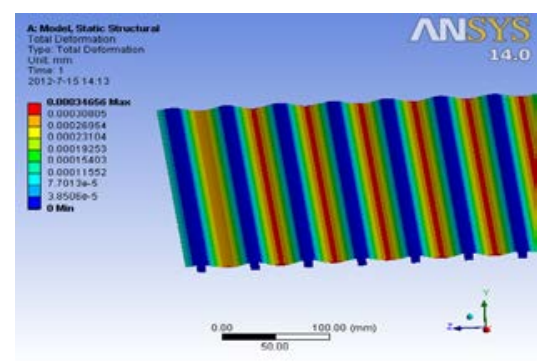

FIGURE VI.(E) DEFORMATION ALONG GLASS EDGE.
FIGURE VI.(C) CENTER DISTANCE OF SUPPORTS IS $70 \mathrm{MM}$, THE MAXIMUM DEFORMATION IS $1.6 \mathrm{M}$.

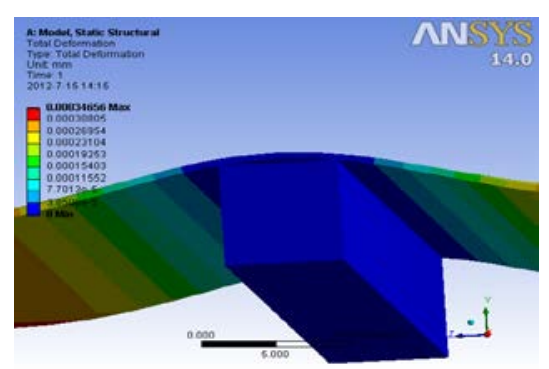

FIGURE VI.(F) DEFORMATION AT THE SUPPORT.

of slotting on the warpage would be. When the slot spacing is $50 \mathrm{~mm}$, the impact of slotting on the warpage is only $0.3 \mu$. 
Taking into account the limits by the granite slotting process, it shall also consider the comprehensive influence of sampling interval and measurement layout of thickness measurement. The slot spacing is $50 \mathrm{~mm}$. The width of supporting edge plane is $5 \mathrm{~mm}$, the slot width is $45 \mathrm{~mm}$, the depth is $3 \mathrm{~mm}$.

\section{SUMMARY}

The spectral confocal sensor is an ideal sensor for the thickness and warpage measurement of TFT substrate glass, in order to accurately measure the thickness and warpage of substrate glass, you should correctly set the conditions for use. Through theoretical analysis and experimental verification, the measurement of spectral confocal sensor should be carried out on the slotted high-precision granite plate. These conclusions have been proved in the TFT substrate glass measurement and successfully applied in engineering.

\section{REFERENCES}

[1] Yang Cheng, WangYutian Glass Thickness measuement system of double Beam path Compensation Based on CPLD Thechnology. Yanshan University

[2] WangXingying,ChangXuguang Instrument of Glass thiekness Measurement, Jounal of transducer Thecknology, 1995, 02 : 29-33.

[3] Song Chen1,Liu Cen,Guo Qi, a princple for Glass Thhickness Measuemrnt Based of Laser Doppler , ACTA PHOTONICA SINICA, 2008, 37 (08) : 1635-1638.

[4] WANG Wei,WANG Zhao-ba, Capability Research of Glass Thickness Detection

[5] Based on CCD Displacement Sensor, Instrument Technique and Sensor, 2006, $9:$ : :44-45.

[6] KATHRYNJ W, Richard R C, GUN Young-Lee. Quantitative in situ measurement of transfer film thickness by a Newton's rings method[J]. Article in Press, 2007, 10(4):6-56.

[7] Maru Yama $H$, Inoue S,Mitsu Yama $T$, et al. Low-coherence interferometer system for the simultaneous measurement of refractive index and thickness[J]. Appl Opt, 2001, 41(3):1315-1322.

[8] Sirat, Gabriel Y., Paz, Freddy, Agronk, Gregory, Wilner, Kalman.Conoscopic holography, SPIE of the International Society for Optical Engineering[J], USA, 2005, 5972: 021-026.

[9] Totzeck, M., Tiziani H.J. Phase-shifting polarization interferometer for microstructure line width measurement[J]. Opt Lett.1999, 24(5): 294-29. 